\title{
ON THE BEST APPROXIMATION OF THE INFINITESIMAL GENERATOR OF A CONTRACTION SEMIGROUP IN A HILBERT SPACE ${ }^{1}$
}

\author{
Elena E. Berdysheva \\ Department of Mathematics, Justus Liebig University Giessen, Germany, \\ elena.berdysheva@math.uni-giessen.de \\ Maria A. Filatova \\ Ural Federal University; \\ Krasovskii Institute of Mathematics and Mechanics, \\ Ural Branch of Russian Academy of Sciences, Ekaterinburg, Russia \\ MA.Filatova@urfu.ru
}

\begin{abstract}
Let $A$ be the infinitesimal generator of a strongly continuous contraction semigroup in a Hilbert space $H$. We give an upper estimate for the best approximation of the operator $A$ by bounded linear operators with a prescribed norm in the space $H$ on the class $Q_{2}=\left\{x \in \mathcal{D}\left(A^{2}\right):\left\|A^{2} x\right\| \leq 1\right\}$, where $\mathcal{D}\left(A^{2}\right)$ denotes the domain of $A^{2}$.
\end{abstract}

Key words: Contraction semigroup, Infinitesimal generator, Stechkin's problem.

\section{Introduction}

Let $H$ be a Hilbert space with the inner product $(\cdot, \cdot)$ and the norm $\|\cdot\|$, and let $A$ be the infinitesimal generator of a strongly continuous contraction semigroup in $H$. For the definition and properties of the infinitesimal generator of a semigroup in a Banach space see, e.g., [6, §14.2]. Note that a strongly continuous contraction semigroup is also called a contraction semigroup of the class $C_{0}([8,9])$. For an operator $F$ on the space $H, \mathcal{D}(F)$ denotes the domain of $F$. We denote by $I$ the identity operator.

In this paper, we study the so-called Stechkin's problem of the best approximation of the operator $A$ by bounded linear operators with a prescribed norm on the class of elements $x \in \mathcal{D}\left(A^{2}\right)$ such that $\left\|A^{2} x\right\| \leq 1$. We give an upper estimate for the best approximation of the operator $A$.

The problem we consider is a special case of the general problem of the best approximation of an unbounded operator by linear bounded ones on a certain class of elements in a Banach space. This problem first appeared in Stechkin's work in 1965-1967 [11]. The problem was studied by a number of authors (see surveys [1], [2], monograph [4], paper [3], and the bibliography therein).

Stechkin formulated this problem in a general setting as follows. Let $X, Y$ be two Banach spaces, let $A$ be a linear operator (in general, unbounded) from $X$ to $Y$, and let $Q \subseteq \mathcal{D}(A)$ be a certain class of elements from the domain $\mathcal{D}(A)$ of the operator $A$. We denote by $\mathscr{B}(N)$ the set of

\footnotetext{
${ }^{1}$ This work was supported by the Russian Foundation for Basic Research (project no. 15-01-02705), the Program for State Support of Leading Scientific Schools of the Russian Federation (project no. NSh9356.2016.1), and by the Russian Academic Excellence Project (agreement no. 02.A03.21.0006 of August 27, 2013, between the Ministry of Education and Science of the Russian Federation and the Ural Federal University).
} 
linear bounded operators from $X$ to $Y$ with the norm $\|T\|_{X \rightarrow Y} \leq N$. The best approximation of the operator $A$ by linear bounded operators $T \in \mathscr{B}(N)$ on the class $Q$ is

$$
E_{N}(A ; Q)=\inf \{U(A, T, Q): T \in \mathscr{B}(N)\},
$$

where

$$
U(A, T, Q)=\sup \left\{\|A x-T x\|_{Y}: x \in Q\right\}
$$

is the deviation of the operator $T$ from the operator $A$ on the class $Q$.

One of the most important cases of the problem formulated above is when the class $Q$ is defined in the following way. Let $Z$ be a Banach space and $B$ be a linear operator from $X$ to $Z$ such that $\mathcal{D}(B) \subseteq \mathcal{D}(A)$. The class $Q$ is then defined as $Q=\left\{x \in X:\|B x\|_{Z} \leq 1\right\}$.

Stechkin [11] suggested an estimate from below for the best approximation $E_{N}(A ; Q)$ in terms of the modulus of continuity of the operator $A$ on the class $Q$ defined by

$$
\Phi(\delta)=\sup \left\{\|A x\|_{Y}: x \in Q,\|x\|_{X} \leq \delta\right\}, \quad \delta>0 .
$$

Namely, Stechkin showed that

$$
E_{N}(A ; Q) \geq \sup \{\Phi(\delta)-N \delta: \delta>0\} .
$$

In particular, when $B=A^{n}$, the problem $E_{N}\left(A^{k} ; Q\right)$ turned out to be closely connected to the exact constants in the Kolmogorov-type inequalities of the form

$$
\left\|A^{k} x\right\| \leq C\|x\|^{\frac{n-k}{n}}\left\|A^{n} x\right\|^{\frac{k}{n}}, \quad x \in \mathcal{D}\left(A^{n}\right),
$$

with $n, k \in \mathbb{N}, 0<k<n$, and a certain constant $C$ that depends on $n$ and $k$.

If $A$ is the differentiation operator, inequalities (1.2) are inequalities between the norms of the derivatives of a function. Such inequalities have been studied by a large number of authors (see [1], [2], [4] and the bibliography therein). Here we only mention that Hardy, Littlewood and Pólya [7, Chapter VII, §7.8] obtained the exact inequality

$$
\left\|f^{\prime}\right\|^{2} \leq 2\|f\|\left\|f^{\prime \prime}\right\|
$$

in the space $L_{2}(0, \infty)$ on the class of functions $f \in L_{2}(0, \infty)$ such that $f^{\prime}$ is locally absolutely continuous on $(0, \infty)$, and $f^{\prime \prime} \in L_{2}(0, \infty)$.

In 1971, Kato [9] proved the following result which can be considered as a generalization of (1.3). Let $A$ be the infinitesimal generator of a strongly continuous contraction semigroup in a Hilbert space $H$. Then

$$
\|A x\|^{2} \leq 2\|x\|\left\|A^{2} x\right\|, \quad x \in \mathcal{D}\left(A^{2}\right) .
$$

In this paper, we study Stechkin's problem of the best approximation of the infinitesimal generator $A$ of a strongly continuous contraction semigroup by bounded linear operators on the class

$$
Q_{2}=\left\{x \in \mathcal{D}\left(A^{2}\right):\left\|A^{2} x\right\| \leq 1\right\}
$$

in a Hilbert space. Namely, we estimate

$$
E_{N}\left(A ; Q_{2}\right)=\inf \{U(T): T \in \mathscr{B}(N)\},
$$

where

$$
U(T)=U\left(A, T, Q_{2}\right)=\sup \left\{\|A x-T x\|: x \in Q_{2}\right\} .
$$




\section{The main result}

The main result of the paper is the following statement.

Theorem 1. The best approximation (1.5) of the infinitesimal generator A of a strongly continuous contraction semigroup in a Hilbert space on the class $Q_{2}$ defined in (1.4) satisfies the inequality

$$
E_{N}\left(A ; Q_{2}\right) \leq \frac{1}{N}
$$

It is known that the infinitesimal generator $A$ of a strongly continuous contraction semigroup in a Banach space possesses the following properties:

1) The domain $\mathcal{D}(A)$ of the operator $A$ is dense (see, e.g., [6, Lemma 14.5, p. 411]).

2) The resolvent set $\rho(A)$ of the operator $A$ contains the right half-plane $\{\lambda \in \mathbb{C} \mid \Re \lambda>0\}$. Moreover, $\left\|(A-\lambda I)^{-1}\right\| \leq(\Re \lambda)^{-1}$ for all $\lambda \in \mathbb{C}$ with $\Re \lambda>0$ (e.g., [6, Theorem 14.7, p. 412]).

Furthermore, if $A$ is the infinitesimal generator of a strongly continuous contraction semigroup in a Hilbert space, we have additionally:

3) The operator $A$ is upper semibounded, with the upper bound 0, i.e.,

$$
\Re(A x, x) \leq 0
$$

for $x \in \mathcal{D}(A)$ [6, Lemma 14.9, p. 416].

The following lemma is not new. However, we will formulate and prove it for the sake of completeness.

Lemma 1. Let $A$ be the infinitesimal generator of a strongly continuous contraction semigroup in a Hilbert space $H$ and $c>0$. Then the operator

$$
B_{c}=(c I+A)(c I-A)^{-1}
$$

is densely defined and bounded (and thus can be extended to the whole space $H$ by continuity). Moreover,

$$
\left\|B_{c}\right\| \leq 1
$$

Remark. The operator $B_{c}$ is the Cayley transform of the operator $A$ in the terminology of Kato [9], see also [10, p. 545].

P r o o f. Since $c>0$, the operator $(c I-A)^{-1}$ is defined everywhere on $H$ and bounded. Since $A$ is the infinitesimal generator of a strongly continuous contraction semigroup, the operator $-A$ is $m$-accretive (see [10, Chapter IX, $\S 1.4$ as well as Problem 1.18, both p. 485]). Therefore, the domain $\mathcal{D}(A)$ of the operator $A$ is equal to the range $\mathcal{R}\left((c I-A)^{-1}\right)$ of the operator $(c I-A)^{-1}$ which is dense in $H$ (see $\left[10\right.$, Chapter V, $\S 3.10$, p. 279]). Thus, $B_{c}$ is densely defined.

Now we estimate the norm of $B_{c}$. For $x \in \mathcal{D}(A)$ we have

$$
\|c x+A x\|^{2}=c^{2}\|x\|^{2}+\|A x\|^{2}+2 c \Re(A x, x),
$$




$$
\|c x-A x\|^{2}=c^{2}\|x\|^{2}+\|A x\|^{2}-2 c \Re(A x, x) .
$$

It follows immediately that

$$
\|(c I+A) x\| \leq\|(c I-A) x\| .
$$

Now take $y \in \mathcal{D}\left((c I-A)^{-1}\right)$. Applying (2.1) to $x=(c I-A)^{-1} y \in \mathcal{D}(A)$, we obtain

$$
\left\|(c I+A)(c I-A)^{-1} y\right\| \leq\|y\|
$$

and thus $\left\|B_{c}\right\| \leq 1$.

Now we are ready to prove Theorem 1.

$\mathrm{P}$ r o o f. We will construct a concrete approximating operator $T$ in problem (1.5) and estimate its norm and its deviation (1.6) from the operator $A$ on the class $Q_{2}$.

Note that all the operators we consider commute on the set $\mathcal{D}\left(A^{2}\right)$.

The restriction of the operator $A$ to the set $\mathcal{D}\left(A^{2}\right)$ (which we will denote by the same symbol) can be represented as

$$
A=\frac{N}{2}\left(B_{N}-I\right)-\frac{1}{2 N}\left(B_{N}+I\right) A^{2}
$$

Put $T: H \rightarrow H$,

$$
T=\frac{N}{2}\left(B_{N}-I\right)
$$

Then, for the restriction of the operator $A-T$ to $\mathcal{D}\left(A^{2}\right)$, we have

$$
A-T=-\frac{1}{2 N}\left(B_{N}+I\right) A^{2} .
$$

We estimate the norm of the operator $T$ as follows:

$$
\|T\|=\frac{N}{2}\left\|B_{N}-I\right\| \leq \frac{N}{2}\left(\left\|B_{N}\right\|+\|I\|\right)=N .
$$

For the deviation $U(T)$ of the operator $T$ from the operator $A$, we obtain that

$$
U(T)=\sup _{x \in Q_{2}}\|(A-T) x\| \leq \sup _{x \in Q_{2}} \frac{1}{2 N}\left\|B_{N}+I\right\| \cdot\left\|A^{2} x\right\| \leq \frac{1}{N} .
$$

It follows immediately from (2.2) and (2.3) that

$$
E_{N}\left(A ; Q_{2}\right) \leq U(T) \leq \frac{1}{N}
$$

\section{Approximation of the differentiation operator in the space $L_{2}(0, \infty)$}

An important concrete case of problem (1.5) is the problem of the best approximation of the differentiation operator $D f=f^{\prime}$ by bounded linear operators in the Hilbert space $L_{2}(0, \infty)$ of real-valued functions whose squares are integrable on $(0, \infty)$ on the class $Q^{(2)}$ defined as follows: 
$Q^{(2)}$ is the class of functions $f \in L_{2}(0, \infty)$ such that $f^{\prime}$ is locally absolutely continuous on $[0, \infty)$, $f^{\prime \prime} \in L_{2}(0, \infty)$, and $\left\|f^{\prime \prime}\right\| \leq 1$. Problem (1.5) takes in this case the form

$$
E_{N}\left(D ; Q^{(2)}\right)=\inf _{T \in \mathscr{B}(N)} \sup _{f \in Q^{(2)}}\left\|f^{\prime}-T f\right\| .
$$

It took about 20 years of research to solve the problem completely. Stechkin's inequality (1.1) and inequality (1.3) of Hardy, Littlewood and Pólya provide the lower bound

$$
E_{N}\left(D ; Q^{(2)}\right) \geq \frac{1}{2 N} .
$$

One of the first upper bounds for (3.1)

$$
E_{N}\left(D ; Q^{(2)}\right) \leq \frac{1}{\sqrt{3} N}
$$

was obtained by using a concrete approximating operator by the first named author in 1996 [5]. Problem (3.1) was fully solved only in 2014 by Arestov and the second named author [3] . Namely, they showed that

$$
E_{N}\left(D ; Q^{(2)}\right)=\frac{1}{2 N} .
$$

In this section, we discuss what the statement of Theorem 1 means in the concrete case (3.1) of problem (1.5). The approximating operator $T$ used in Theorem 1 is

$$
T=\frac{N}{2}\left(B_{N}-I\right)=N A(N I-A)^{-1} .
$$

Below we will describe this operator in the special case. We consider and calculate its norm $\|T\|$ and its deviation $U(T)$ from the operator $A=D$ on the class $Q^{(2)}$.

It is not difficult to see that the operator $T$ in the concrete case can be represented as follows. Let $W$ be the class of functions $y \in L_{2}(0, \infty)$ such that $y$ is locally absolutely continuous on $[0, \infty)$ and $y^{\prime} \in L_{2}(0, \infty)$. For $f \in L_{2}(0, \infty)$, we consider the differential equation

$$
-y^{\prime}+N y=f, \quad y \in W .
$$

For each function $f \in L_{2}(0, \infty)$, equation (3.3) has a unique solution which is a real-valued function from $L_{2}(0, \infty)$. The operator $T$ is defined as

$$
T f=N y^{\prime},
$$

where $y$ is the solution of the differential equation (3.3).

Integrating by parts and taking into account that $\lim _{t \rightarrow \infty} y(t)=0$, we obtain (see [3] for details) that

$$
\|f\|^{2}=\int_{0}^{\infty}\left(-y^{\prime}(t)+N y(t)\right)^{2} d t=\int_{0}^{\infty}\left(y^{\prime}(t)\right)^{2} d t+N^{2} \int_{0}^{\infty}(y(t))^{2} d t+N y^{2}(0) .
$$

It follows from (3.4) that $\|T f\|^{2}=N^{2} \int_{0}^{\infty}\left(y^{\prime}(t)\right)^{2} d t$. Thus, we immediately obtain

$$
\|T f\|^{2} \leq N^{2}\|f\|^{2}
$$


which gives the estimate $\|T\| \leq N$. Now we show that indeed $\|T\|=N$. Consider the family of functions $y_{K}=e^{-K t}, K>0$. Let $f_{K}$ be the corresponding right-hand side of equation (3.3). Take an arbitrary $0<\alpha<1$. We have

$$
\begin{aligned}
\alpha N^{2}\left\|f_{K}\right\|^{2}-\left\|T f_{K}\right\|^{2}= & \alpha N^{2} \int_{0}^{\infty}\left(-y_{K}^{\prime}(t)+N y_{K}(t)\right)^{2} d t-N^{2} \int_{0}^{\infty}\left(y_{K}^{\prime}(t)\right)^{2} d t \\
& =\frac{N^{2}}{2 K}\left(\alpha(K+N)^{2}-K^{2}\right) .
\end{aligned}
$$

This expression is negative for all $0<\alpha<\frac{K^{2}}{(N+K)^{2}}$ which yields $\left\|T f_{K}\right\|^{2}>\alpha N^{2}\left\|f_{K}\right\|^{2}$. Letting $K$ go to infinity (with fixed $N$ ) we let $\alpha$ approach 1 , and thus obtain $\|T\| \geq N$. Consequently, $\|T\|=N$.

Note that inequality (3.5) is a strict inequality if $y \neq 0$ and, consequently, $f \neq 0$. In other words, the norm of the operator $T$ is not attained.

It can be shown similarly that the norm of the operator $V=-\frac{1}{2 N}\left(B_{N}+I\right)$ is equal to $1 / N$. Since the domain $\mathcal{D}\left(D^{2}\right)$ of the operator $D^{2}$ is dense in $L_{2}(0, \infty)$, it follows that the deviation of the operator $T$ from the differentiation operator $D$ on the class $Q^{(2)}$ is equal to $1 / N$.

Thus, the approximating operator (3.2) gives the estimate $E_{N}\left(D ; Q^{(2)}\right) \leq \frac{1}{N}$ in the general case (1.5) as well as in the concrete case (3.1).

\section{REFERENCES}

1. Arestov V.V. Approximation of unbounded operators by bounded operators and related extremal problems // Russian Math. Surveys, 1996. Vol. 51, no. 6. P. 1093-1126. DOI: 10.4213/rm1019

2. Arestov V.V., Gabushin V.N. Best approximation of unbounded operators by bounded operators // Russian Math. (Iz. VUZ), 1995. Vol. 39, no. 11. P. 38-63.

3. Arestov V.V., Filatova M.A. Best approximation of the differentiation operator in the space $L_{2}$ on the semiaxis // J. Approx. Theory, 2014. Vol. 187. P. 65-81. DOI: 10.1016/j.jat.2014.08.001

4. Babenko V.F., Korneichuk N.P., Kofanov V.A., Pichugov S.A. Inequalities for derivatives and their applications. Kiev: Naukova Dumka, 2003. [in Russian]

5. Berdysheva E.E. On the best approximation of the differentiation operator in $L_{2}(0, \infty) / /$ East J. Approx. 1996. Vol. 2, no. 3. P. 281-287.

6. Grubb G. Distributions and Operators. NY: Springer Science and Business Media, 2008. 464 p. DOI: $10.1007 / 978-0-387-84895-2$

7. Hardy G.H., Littlewood J.E., Pólya G. Inequalities. Cambridge University Press, 1934. 314 p.

8. Hille E., Phillips R.S. Functional Analysis and Semigroups. American Mathematical Society, 1957. $808 \mathrm{p}$.

9. Kato T. On an inequality of Hardy, Littlewood and Pólya // Adv. Math., 1971. Vol. 7. P. 217-218. DOI: $10.1016 /$ S0001-8708(71)80002-6

10. Kato T. Perturbation Theory for Linear Operators. Verlag-Berlin-Heidelberg: Springer, 1995. 623 p. DOI: $10.1007 / 978-3-642-66282-9$

11. Stechkin S.B. Best approximation of linear operators // Math. Notes. 1967. Vol. 1. P. 91-99. DOI: $10.1007 / \mathrm{BF} 01268056$ 